\title{
Discussion on the Pathogenesis of Hemorrhoids
}

\author{
Siyuan Liu' ${ }^{1}$ Junming Hou' ${ }^{2}$, Wenzhe Feng², Dezhen Yang2 ${ }^{2}$ Lu Luo $^{2}$ \\ ${ }^{1}$ Shaanxi University of Traditional Chinese Medicine, Xixian New District 712046, Shaanxi Province, China \\ ${ }^{2}$ The Affiliated Hospital of Shaanxi University of Traditional Chinese Medicine, Xianyang City, 712000, Shaanxi Province, \\ China
}

*Corresponding author: Junming Hou, houjunming078@126.com

\begin{abstract}
With the efficient development of social economy, as well as the acceleration of the pace of life, the incidence of hemorrhoids remains high all year round, occupying a relatively high incidence of anorectal diseases. The author will sort out the four most representative theories of pathogenesis, and summarize and analyze them. Understanding the pathogenesis of hemorrhoids can give a deeper understanding of the nature of the disease, and it will help improve the accuracy of clinical diagnosis and treatment, which in turn is beneficial to the improvement of the cure rate.
\end{abstract}

Keywords: Hemorrhoids; Pathogenesis; Preventive treatment

Publication date: July 2021; Online publication: July 31, 2021

\section{Introduction}

With the efficient development of social economy, as well as the acceleration of the pace of life, the incidence of hemorrhoids remains high all year round, in anorectal diseases, the incidence rate is as high as $87.25 \%{ }^{[1]}$. It has significant clinical features such as blood, prolapse, swelling and pain, and can be divided into three types: internal hemorrhoids, external hemorrhoids and mixed hemorrhoids according to the positional relationship between the diseased part and the anal canal tooth line. Understanding the pathogenesis of hemorrhoids can give a deeper understanding of the nature of the disease, and it will help improve the accuracy of clinical diagnosis and treatment, which in turn is beneficial to the improvement of the cure rate. The author will sort out the four most representative theories of pathogenesis, summarize and analyze them, and elaborate on them as follows.

\section{Four pathogenesis theories of hemorrhoids}

The occurrence of hemorrhoids is closely related to its own anatomical structure, tissue composition and physiological characteristics. The female hemorrhoid area of the human body is also known as anal cushion. The full name is vascular cushion. It is divided into three locations on the left, right front and right back of the anus, namely at 3, 7, and 11 points of the lithotomy position. The blood supply is abundant, the arteries are composed of the superior and inferior rectal arteries, the anal artery and the middle sacral artery, and the veins are composed of the superior and inferior rectal venous plexus. The direct anastomosis between the arteries and veins is called sinus veins, and this type of blood vessel pattern becomes a distinctive feature of the hemorrhoid area. Treitz muscle is a complex of connective tissue and smooth muscle in a networklike structure ${ }^{[2]}$. The anal cushion tissue can be fixed to the internal anal sphincter by wrapping the rectal venous plexus to prevent it from shifting and sagging. A full understanding of the above-mentioned related concepts helps to better understand the pathogenesis of hemorrhoids. 


\subsection{The theory of downward movement of the anal cushion}

Thomson first pointed out in 1975 that "anal cushion" is a complex composed of blood vessels, connective tissue and smooth muscle fibers. Anal cushion is a normal anatomical structure located in the anal canal, formed by the fetus differentiation in the mother's body. Hemorrhoids are pathological hypertrophy of the anal pad ${ }^{[2]}$. It cooperates with the sphincter to achieve the effect of closing the anus. In 1994, the "anal cushion downward shift theory" was proposed that when physiological functions are functioning normally, the anal cushion is attached to the wall of the internal sphincter and uses the contractility of its muscle fibers to restore the downward displacement due to excretion. When the function of the Treitz muscle used for fixation is weakened or lost for some reason, it will induce congestion and swelling of the anal cushion, downward movement and even out of the body, causing hemorrhoids. Under the investigation of scholar Zhenjun Wang and others, the morphological differences between hemorrhoids and normal anal cushions were compared, the Treitz muscle fibers and connective tissues of the former are disorderly and loosely arranged, with significant deformation and interruption, while the latter is regular and aggregated. There are few morphological changes ${ }^{[3]}$.

\subsection{Theory of varicose veins}

This theory has a long history. In 1960, the "varicose vein theory" was first put forward ${ }^{[4]}$. This theory believes that the formation of hemorrhoids is caused by varicose veins in the anorectal canal. Due to the vein in this place lacks a venous valve similar to the portal vein, the arteries in the hemorrhoid area are easily compressed when passing through the surrounding muscles, which hinders the return of venous blood, congestion in the vessel over time, and the expansion and tortuosity of the vessel wall to form a venous cluster is hemorrhoids. Anatomical evidence suggests that varicose rectal venous plexus can be found in the opened hemorrhoids.

\subsection{Theory of vascular proliferation}

At the beginning of the 19th century, some people put forward the term "rectal cavernous body," which became the origin of the theory of vascular hyperplasia. This theory believes that there is tissue hyperplasia under the epidermis of the anal canal, which is similar to the erectile tissue, composed of sinus-shaped veins under the anal canal. The tube wall has many glial fibers, and the muscle layer is underdeveloped. It is prone to congestion, swelling and hyperplasia, resulting in hemorrhoids. Han Wei scholars have made a histological comparison between normal anal cushions and hemorrhoids, and the results showed that the density of micro-vessels in hemorrhoids was significantly increased, and new blood vessels were formed ${ }^{[5]}$. The correlation between vascular proliferation and hemorrhoid formation was confirmed.

\subsection{Theory of degeneration of hemorrhoids}

In 2014, Professor Xiangdong Yang from Chengdu University of Traditional Chinese Medicine (TCM) creatively put forward the viewpoint of "hemorrhoid degeneration theory." The theory believes that hemorrhoids are degenerative changes of blood vessels and tissues in the terminal rectum, that is, aging and degeneration ${ }^{[6]}$. The blood vessels, muscle fibers, and connective tissues of the anorectum are onedimensional just like the passage of time. As people age and other factors, the blood vessels become thinner and loose, and hemodynamic changes occur, which affect the return of blood and veins. Vascular flexion and hyperplasia form venous masses; muscle fibers and connective tissues become loose or even torn, resulting in separation of rectal mucosa and muscles, and downward displacement of the anal cushion, causing hemorrhoids. This theory is consistent with the senescence and degeneration of cells in the course of biological life.

In the clinical and research fields of modern medicine, the first three theories are widely recognized 
because they were put forward earlier. The theory of lower anal cushion emphasizes tissue degenerative changes, the theory of vascular proliferation and varicose veins emphasize the changes of blood vessels and hemodynamics, and the theory of degeneracy considers both tissue and vascular degenerative changes comprehensively, compared with the first three There is a more complete explanation of the onset of hemorrhoids.

\section{Conclusion}

Hemorrhoids is a common clinical disease, and the incidence rate remains high all year round. This is determined by the modern lifestyle and the aging of the global population. Although the onset of hemorrhoids is generally not life-threatening, its symptoms and complications can easily affect the patient's living conditions, and even severely hinder the patient's normal life. In the long run, it will adversely affect the patient's mental health and further aggravate the symptoms. This vicious cycle will eventually damage personal physical and mental health. Therefore, understanding the pathogenesis of hemorrhoids plays an important role in clinical treatment, especially prevention. TCM emphasizes "preventing the disease," fully clearing the ins and outs of the onset of hemorrhoids, applying theories to prevention, and doctors and patients cooperate with each other, and it will be better to prevent the disease before the disease, prevent the change of the existing disease, accurately treat and prevent recurrence. To control the disease locally, to ensure the physical and mental health of the people, and to lay another cornerstone for the development of TCM in China.

\section{Disclosure statement}

The author declares no conflict of interest.

\section{References}

[1] 2020, Professional Committee of Colorectal Diseases, Chinese Society of Integrated Traditional Chinese and Western Medicine. Guidelines for Diagnosis and Treatment of Hemorrhoids in China (2020). Colorectal and Anal Surgery, 26(05):519-533.

[2] Yang Y, Yang X, 2018, The Pathophysiology of Hemorrhoid Degeneration Theory. Chinese Journal of Burns Wounds \& Surface Ulcers, 30(05):378-380.

[3] Wang Z, Tang X, Wang D, et al., 2006, The Pathological Features and Significance of Internal Hemorrhoids. Chinese Journal of Surgery, (03):177-180.

[4] Scott RS, Tracy LH, Thomas ER, et al., 2016, The ASCRS Textbook of Colon and Rectal Surgery. Springer, Cham.

[5] Han W, Wang Z, Zhao B, et al., 2005, The Mechanism and Significance of Elastic Fiber Degeneration and Angiogenesis in Hemorrhoid Tissue. Chinese Journal of Gastrointestinal Surgery, (01):56-59.

[6] Chen Q, Yang X, 2014, Theory and Application of Degeneration of Hemorrhoids. Colorectal and Anal Surgery, 20(06):435-436. 